\title{
Numerical solution for the Schrödinger equation with potential in graphene structures
}

\author{
L. A. Nhat ${ }^{1,2}$ \\ ${ }^{1}$ Peoples' Friendship University of Russia (RUDN University), \\ 6 Miklukho-Maklaya str., 117198, Moscow, Russia \\ ${ }^{2}$ Tan Trao University, 22227, Tuyen Quang, Vietnam \\ leanhnhat@mail.ru
}

DOI 10.17586/2220-8054-2019-10-2-124-130

\begin{abstract}
This paper presents a different numerical solution to compute eigenvalues of the Schrödinger equation with the potentials in graphene structures [1]. The research subjects include the Schrödinger equation and the exchange-correlation energy of the graphene structures in Grachev's article. Specifically, we used the pseudospectral method basing on the Chebyshev-Gauss-Lobatto grid to determine the approximate numerical results of the problem. The results are the discrete energy spectra and the corresponding eigenfunctions of the nonlinear spin waves in the graphene structure. Additionally, these results can be applied to create the nonlinear spin waves in the graphene structures.
\end{abstract}

Keywords: graphene, kinks, breathers, spin, pseudospectral method, Schrödinger equation, Chebyshev, eigenvalue problems, nonlinear models.

Received: 12 April 2019

Revised: 17 April 2019

\section{Introduction}

In 2010, D. D. Grachev and L. A. Sevastyanov invented the method for generating spin waves [2,3]. This method formed the quantum collective excitations of spin density and magnetization density in graphene films. It may be used in quantum nanoelectronics, spintronics, for creating spin-processors, memory cells, physical field sensors, other devices and systems for processing and storing information of terahertz (and higher) range that have nanometric dimensions and work in a broad temperature range with minimum energy consumption.

Since the publication of this invention, the authors studied, built and perfected the theory of the method for generating spin waves. In particular, Grachev constructed the quantum field model to adequately describe ferromagnetic properties in graphene structures to match the results of physical and numerical experiments. This model described properties of monoatomic graphene layers, which connected with the presence of a nontrivial function of the distribution of the spin density, formed as a result of the spontaneous breakdown of the spin symmetry of valence electrons in atoms of carbon [4]; in [5], the authors provided the nonlinear field model to describe of the spin density distribution of the valence electrons in graphene films. This model describes experimentally observable ferromagnetic properties of such films; prior work [6] claimed that the offered variant of the nonlinear field model, in which carriers of spin density are not fermions (electrons), but bosons (spinons), is quite adequate for describing the magnetic properties of graphene structures. The authors proposed in two articles $[1,7]$ the desirability of a nonlinear model that describes a possible mechanism of ferromagnetism in graphene structures, resulting from electron-electron interaction and spontaneous breaking of spin symmetry of valence electrons. We investigated such spatially localized nonlinear spin of the valence electron density on the graphene surface such as kinks, and their interactions, as well as quasibound metastable states of the interacting kinks and antikinks, that are breathers [8].

In addition, many scientists have also studied how to spin waves in graphene, and they have gained certain achievements such as the excitations with spin reversal such as spin-flip and spin-wave excitations were studied in [9]. They showed that these excitations were correctly accounted for in the time-dependent Hartree-Fock and strong magnetic field approximations; F. J. Culchac investigated spin excitations and electronic properties of graphene nanoribbon devices with zigzag edges [10,11]. Those studies showed that a central point of a finite zigzag nanoribbon, when spin excitations are damped at all finite energies, their energy dispersion at small wave vector is dominated by antiferromagnetic correlations between the ribbons edges, in accordance with previous calculations. A. Matthew studied the collective excitations of doped graphene in the presence of in-plane magnetic fields and calculated the dispersions of charge and spin plasmons using time-dependent density-functional methods within a standard tight-binding approach [12].

Evidently, in the articles [1,4-8], it is shown that the density of spin symmetry was broken by the spontaneous breaking, which obeyed a nonlinear equation, and the offered nonlinear models with the limits will exist exact and 
approximate solutions for the distribution of the spin density and magnetization on the surface of the graphene structures, as the method of the scattering matrix [5]; the finite element method (FEM) and the Ritz method [1,7]; the Ritz method using Hermitian functions as coordinate functions [8].

Based on the density functional theory and the perturbation methods, in [1] proposed a physically reasonable nonlinear model of interacting massless Dirac fermions in graphene structures for practical applications. This model described the spin density $(s)$ distribution of the valence electrons of the carbon atoms in graphene structures. This corresponds to the equation of the well-known nonlinear model $\left(\lambda \phi^{4}\right)$ :

$$
s^{\prime \prime}=\lambda\left(s^{2}-s_{0}^{2}\right) s,
$$

where $s_{0}$ - the zero-order local spin density, $\lambda$ - the self-interaction constant.

To illustrate this, consider the simplest nonlinear model $\lambda \phi^{4}$ when the envelopes depend on one spatial coordinate. It is well known that the Hamiltonian density of the model has the form:

$$
H[s]=\left(\partial_{\nu} s \partial^{\nu} s\right) / 2+\lambda\left(s^{2}-s_{0}^{2}\right) / 4, \quad \nu=0,1,2 .
$$

In this case, equation (1) was known to have two stable vacuum solutions: $s_{ \pm}= \pm s_{0}$ and the kink-antikink solutions:

$$
s_{ \pm}= \pm s_{0} t h\left(\sqrt{\frac{\lambda s_{0}^{2}}{2} x}\right) .
$$

Due to the non-linearity of equation (1), for qualitative estimates, the author determined approximate solutions of equation (1) by choosing the field function of interacting kinkantikink pair in such simple form:

$$
\Phi(x, a)=s_{+}(x+a)+s_{-}(x-a)-s_{0}
$$

where $a$ is the parameter, and the function (3) has the following asymptotic behavior:

$$
\left\{\begin{array}{c}
\Phi(x,+\infty)=+s_{0} \\
\Phi(+\infty, a)=\Phi(-\infty, a)=-s_{0} \\
\Phi_{x}^{\prime}(+\infty, a)=\Phi_{x}^{\prime}(-\infty, a)=0 \\
\Phi(x,-\infty)=-3 s_{0}
\end{array}\right.
$$

or

$$
\left\{\begin{array}{c}
\Phi(x,+\infty)=+s_{0}, \\
\Phi(+\infty, a)=\Phi(-\infty, a)=-s_{0} \\
\Phi_{x}^{\prime}(+\infty, a)=\Phi_{x}^{\prime}(-\infty, a)=0 \\
\Phi(x, 0)=-s_{0} ;
\end{array}\right.
$$

It can easily be seen that there are two models. We call the conditions (4) - model A, and the conditions (5) - model B.

We can write a system of the Hamiltonian with the field function in the form (3) that satisfies the equation of the type $(1)[1,7]$ :

$$
H\{\Phi, a\}=\frac{1}{2} \int_{-\infty}^{\infty} d x\left\{\left[\Phi_{x}^{\prime}(x, a)\right]^{2}+\frac{\lambda}{2}\left[\Phi(x, a)^{2}-s_{0}^{2}\right]^{2}\right\} .
$$

Similarly, we can write the energy density of the kink [7]:

$$
E[s]=\int_{-\infty}^{\infty} d x\left\{\frac{s_{x}^{\prime 2}}{2}+\frac{\lambda}{2}\left[s^{2}-s_{0}^{2}\right]^{2}\right\} .
$$

Combining (7) and (2) gives the result:

$$
E[s]=\frac{2 \sqrt{2 \lambda}}{3} s_{0}^{3}
$$

Then, the sum of the mass-energy equivalent of free kink and antikink equals the potential energy of the breather depending on $a$ with $U\{\Phi, a\}$ computed from (6) and the function $H\{\Phi, a\}$ possesses a minimum. We have the mass of the breather:

$$
m\left[\lambda, s_{0}\right]=\frac{4 \sqrt{2 \lambda}}{3} s_{0}^{3}
$$


The Schrödinger equation for the quantum-mechanical wave function $\psi(a)$ of a stationary breather state with corresponding eigenenergy $E$ has the form:

$$
-\frac{\hbar^{2}}{2 \widetilde{m}\left\{\lambda, s_{0}\right\}} \frac{\mathrm{d}^{2}}{\mathrm{~d} a^{2}} \psi(a)+U(\Phi, a) \psi(a)=E \psi(a),
$$

here $\widetilde{m}\left\{\lambda, s_{0}\right\}=m\left\{\lambda, s_{0}\right\} / c^{2}$ is the effective mass of the breather, $c$ is the speed of light, $U(\Phi, a)$ is the potential energy, $\psi(a)$ is the wave function and $E$ is the energy of the system.

Grachev's articles used the finite element method (FEM) and the conventional Ritz method to determine the eigenvalues and eigenfunctions of the Schrödinger equation (10) for the quantum-mechanical wave.

In this paper, we study the numerical solution to compute eigenvalues of the Schrödinger equation (10) with the energies of the system in graphene structures. That is the pseudospectral method basing on the Chebyshev polynomials of the first kind and using the Chebyshev-Gauss-Lobatto grid in the integration interval $[-1,1]$.

\section{Chebyshev differentiation matrix (CDM)}

A grid function $v(x)$ is defined on the Chebyshev-Gauss-Lobatto points $x=\left\{x_{0}, x_{1}, \ldots, x_{n}\right\}$ such that $x_{k}=\cos (k \pi / n), k=\overline{0, n}$. They are the extrema of the $n$-th order in the Chebyshev polynomial $T_{n}(x)=$ $\cos \left(n \cos ^{-1} x\right)$. The function $v(x)$ is interpolated by constructing the $n$-th order interpolation polynomial $g_{j}(x)$ such that $g_{j}\left(x_{k}\right)=\delta_{j, k}$,

$$
p(x)=\sum_{j=0}^{n} p_{j} g_{j}(x)
$$

where $p(x)$ is the unique polynomial of degree $n$ and $p_{j}=v\left(x_{j}\right), j=\overline{0, n}$. The following can be shown:

$$
g_{j}(x)=\frac{(-1)^{j+1}\left(1-x^{2}\right) T_{n}^{\prime}(x)}{c_{j} n^{2}\left(x-x_{j}\right)}, \quad j=\overline{0, n},
$$

where

$$
c_{j}=\left\{\begin{array}{lc}
2, & j=0 \text { or } n, \\
1, & \text { otherwise. }
\end{array}\right.
$$

As we know the values of $p(x)$ at $n+1$ points, we would like to find approximately the values of the derivative of $p(x)$ at those points $p^{\prime}(x)=\frac{d}{d x} p(x)$. We can write the same in the matrix form:

$$
p^{\prime}=D p
$$

where $D=\left\{d_{i, j}^{(1)}\right\}$ is an $(n+1) \times(n+1)$ differentiation matrix.

Evidently, the derivative of $p\left(x_{j}\right)$ becomes:

$$
p^{\prime}\left(x_{j}\right)=\sum_{k=0}^{n} D_{j, k} p\left(x_{k}\right), \quad j=\overline{0, n} .
$$

We have the entries $d_{i, j}^{(1)}=g_{i}^{\prime}\left(x_{j}\right)$ which are $[13,14]$

$$
\begin{gathered}
d_{0,0}^{(1)}=-d_{n, n}^{(1)}=\frac{2 n^{2}+1}{6}, \\
d_{i, i}^{(1)}=-\frac{x_{i}}{2\left(1-x_{i}^{2}\right)}, \quad i=\overline{1, n-1} \\
d_{i, j}^{(1)}=\frac{c_{i}}{c_{j}} \frac{(-1)^{i+j}}{x_{i}-x_{j}}, \quad i \neq j, \quad i, j=\overline{1, n-1}
\end{gathered}
$$

where $c_{k}$ is determined by the formula (13).

Similarly, $p^{\prime}(x)$ is a polynomial of degree $n-1$; it exists the second differentiation matrix $D^{2}$,

$$
p^{\prime \prime}=D^{2} p
$$

and

$$
p^{\prime \prime}\left(x_{j}\right)=\sum_{k=0}^{n} D_{j, k}^{2} p\left(x_{k}\right), \quad j=\overline{0, n}
$$




\section{Pseudospectral method using the CDM for the Schrödinger equation}

Suppose the Schrödinger equation has simple form as the following:

$$
-\frac{d^{2}}{d x^{2}} u(x)+q(x) u(x)+\lambda u(x)=0, \quad u(-1)=u(1)=0
$$

and collocation points $x_{i}$ such that $1=x_{0}>x_{1}>\ldots>x_{n}=-1$.

We know that:

$$
\frac{d^{2}}{d x^{2}} u_{n}\left(x_{i}\right)=\sum_{k=0}^{n} D_{i, k}^{2} u_{n}\left(x_{k}\right)
$$

Thus, equation (19) takes the following form:

$$
-\sum_{k=0}^{n} D_{i, k}^{2} u_{n}\left(x_{k}\right)+q\left(x_{i}\right) u\left(x_{i}\right)=-\lambda u\left(x_{i}\right), \quad i=\overline{1, n-1}
$$

with $u_{n}\left(x_{n}\right)=0$ and $u_{n}\left(x_{0}\right)=0$.

Alternately, we partition the matrix $D$ into matrices $[13,15]$ :

$$
\begin{gathered}
\widetilde{E}^{(1)}=\left(\begin{array}{cccc}
d_{1,1}^{(1)} & d_{1,2}^{(1)} & \cdots & d_{1, n-1}^{(1)} \\
d_{2,1}^{(1)} & d_{2,2}^{(1)} & \cdots & d_{2, n-1}^{(1)} \\
\vdots & \vdots & \ddots & \vdots \\
d_{n-1,1}^{(1)} & d_{n-1,2}^{(1)} & \cdots & d_{n-1, n-1}^{(1)}
\end{array}\right), \\
\widetilde{e}_{0}^{(1)}=\left(\begin{array}{c}
d_{1,0}^{(1)} \\
d_{2,0}^{(1)} \\
\vdots \\
d_{n-1,0}^{(1)}
\end{array}\right), \widetilde{e}_{n}^{(1)}=\left(\begin{array}{c}
d_{2, n}^{(1)} \\
\vdots \\
d_{n-1, n}^{(1)}
\end{array}\right)
\end{gathered}
$$

We can rewrite in the matrix form: $\widetilde{e}_{0}^{(1)}=\left\{d_{i, 0}^{(1)}\right\}, \widetilde{E}^{(1)}=\left\{d_{i, j}^{(1)}\right\}, \widetilde{e}_{n}^{(1)}=\left\{d_{i, n}^{(1)}\right\}$, here $i, j=\overline{1, n-1}$.

Similarly, we partition matrix $D^{2}$ into matrices $\widetilde{e}_{0}^{(2)}, \widetilde{E}^{(2)}, \widetilde{e}_{n}^{(2)}$. So, (21) can then be written in the matrix form:

$$
-\left(u_{n}\left(x_{0}\right) \widetilde{e}_{0}^{(2)}+\widetilde{E}^{(2)} u+u_{n}\left(x_{n}\right) \widetilde{e}_{n}^{(2)}\right)+Q u=-\lambda u .
$$

Since $u_{n}\left(x_{0}\right)=0$ and $u_{n}\left(x_{n}\right)=0$, we have derived the following:

$$
\left(-\widetilde{E}^{(2)}+Q\right) u=-\lambda u
$$

where $u$ denotes the vectors with elements $\left\{u_{n}\left(x_{i}\right)\right\}$, while $Q$ denotes the diagonal matrix with elements $\left\{g\left(x_{i}\right)\right\}, i=\overline{1, n-1}$.

\section{Numerical solutions and results}

In our research, the program has used the Mathematica 10.4 language [16]. Our numerical results are computed by the pseudospectral method using the CDM (CPSM), as shown in the CPSM columns. These numerical results as per Grachev's article have been included in the columns FEM for the sake of completeness.

The potential energy $U(\Phi, a)$ in nonlinear spin waves in graphene structures has been calculated; it had the analytic form $[1,7]$ :

$$
U\{\Phi, a\}=\frac{4 \sqrt{2 \lambda}}{3} s_{0}^{3}\left(1-\frac{6}{t}+\frac{36 \sqrt{2 \lambda} s_{0} a-24}{t^{2}}+\frac{48 \sqrt{2 \lambda} s_{0} a}{t^{3}}\right)
$$

where

$$
t= \begin{cases}e^{2 \sqrt{2 \lambda} s_{0} a}-1 & \text { for model A } \\ e^{2 \sqrt{2 \lambda} s_{0}|a|}-1 & \text { for model B }\end{cases}
$$


From section 4 of the article [1], we can deduce the following: if the vicinity of $a=0$ and $|a|<1 /\left(5 s_{0} \sqrt{2 \lambda}\right)$, this potential has the following asymptotic form of a power series:

$$
\begin{aligned}
U(\Phi, a)=8 s_{0}^{5} \lambda \sqrt{2 \lambda} a^{2}\left(\frac{2}{5}-\frac{4 s_{0} \sqrt{2 \lambda}}{15}+\right. & \frac{s_{0}^{2} \lambda a^{2}\left(4+7 s_{0} \sqrt{2 \lambda} a\right)}{105} \\
& \left.-\frac{16 s_{0}^{4} \lambda^{2} a^{4}}{525}-\frac{32 s_{0}^{5} \lambda^{2} \sqrt{2 \lambda} a^{5}}{1575}+\frac{416 s_{0}^{6} \lambda^{3} a^{6}}{3465}+\frac{\left.32 s_{0}^{7} \lambda^{3} \sqrt{2 \lambda} a^{7}\right)}{6237}\right) ;
\end{aligned}
$$

if $a \rightarrow+\infty$ then $\left.U_{\max }=U(\Phi,+\infty)=4 \sqrt{2 \lambda} s_{0}^{3}\right) / 3$; for model A, at $a \rightarrow-\infty$ then potential $U(\Phi,-\infty)=$ $4 \sqrt{2 \lambda} s_{0}^{3}(6|a|-17) / 3$. With $s_{0}=2$ and $\lambda=1$, we have graphs of potential energy $U(\Phi, a)$ in the cases model A (denoted by $U_{A}$ ) and model B (denoted by $U_{B}$ ) as depicted in Fig. 1 .

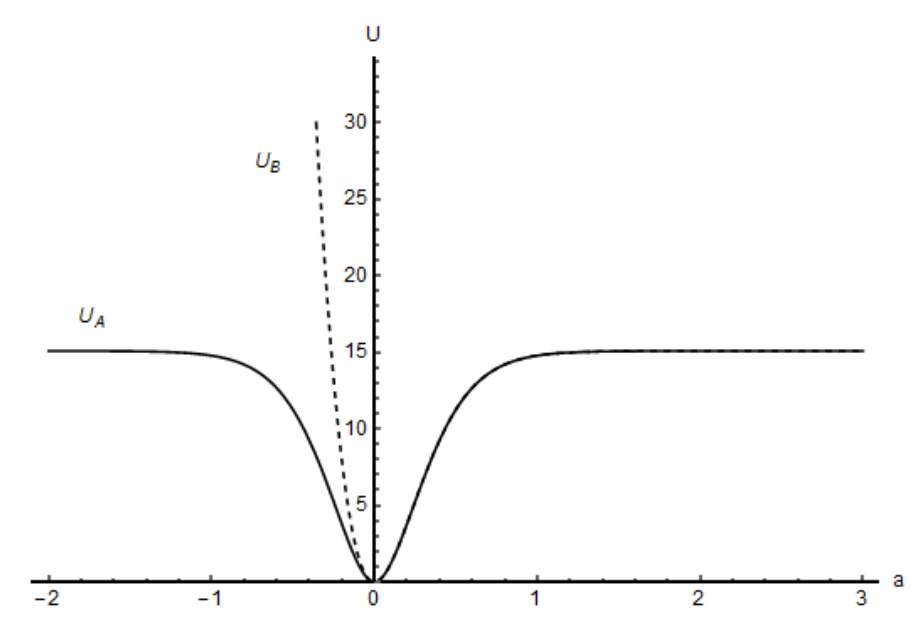

FIG. 1. Two cases model $\mathrm{A}$ and $\mathrm{B}$ of the potential energy $U(\Phi, a)$ in nonlinear spin waves in graphene structures with $s_{0}=2$ and $\lambda=1$

Now, we consider the Schrödinger equation (10) in the case $\hbar=c=1$ :

$$
-\frac{\mathrm{d}^{2}}{\mathrm{~d} a^{2}} \psi(a)+2 \bar{U}(\Phi, a) \psi(a)=2 \bar{E} \psi(a)
$$

here

and

$$
2 \bar{U}(\Phi, a)=2 m\left\{\lambda, s_{0}\right\} U(\Phi, a)=\frac{8 \sqrt{2 \lambda} s_{0}^{3}}{3} U(\Phi, a)
$$

$$
2 \bar{E}=2 m\left\{\lambda, s_{0}\right\} E=\frac{8 \sqrt{2 \lambda} s_{0}^{3}}{3} E .
$$

We apply the section 3 for the equation (27), we can thus rewrite in the matrix form:

$$
\left(-\widetilde{E}^{(2)}+G\right) \psi=2 \bar{E} \psi \text {. }
$$

where $G$ is the diagonal matrix of order $n-1$, with the elements:

$$
\left\{\frac{8 \sqrt{2 \lambda} s_{0}^{3}}{3} U\left(\Phi, a_{i}\right)\right\}, \quad i=\overline{1, n-1} .
$$

Thus, to find the energy $2 \bar{E}$ of the system (27) and find eigenvalues $2 \bar{E}$ in the equation (28) are equivalent, we have to deduce the eigenvalues of matrix $-\widetilde{E}^{(2)}+G$ and the total energy $E=\frac{3 \bar{E}}{4 s_{0}^{3} \sqrt{2 \lambda}}$ as is shown in the Tab.1 for the two case models A (denoted by $E^{A}$ ) and B (denoted by $E^{B}$ ), with $\lambda=1$ and and $s_{0}=2$. Therefore, we have the graphics illustrating the first four eigenfunctions of the breather states of models A (denoted by $\psi^{A}(a)$ ) Fig. 2 and B (denoted by $\psi^{B}(a)$ ) - Fig. 3.

Remarks: From the numerical results in Table 1, we see that: the numerical results of CPSM and FEM are equivalent. In addition, it provides many arbitrary numerical results based on practical applications. It is clear that this numerical solution is reliable and very accurate when $k<2 n / \pi$ [17]. Hence, it may become our calculation 
TABLE 1. First ten eigenvalues of the total energy $E^{A}$ and $E^{B}$ in the cases model $\mathrm{A}$ and $\mathrm{B}$ with $\lambda=1$ and $s_{0}=2$.

\begin{tabular}{|c||c|c|c|c|}
\hline \multicolumn{1}{|c||}{} & \multicolumn{2}{c|}{ CPSM } & \multicolumn{2}{c|}{ FEM } \\
\hline$k$ & $E^{A}$ & $E^{B}$ & $E^{A}$ & $E^{B}$ \\
\hline \hline 1 & 2.118495 & 1.870306 & 2.11 & 1.87 \\
\hline 2 & 6.063236 & 5.232607 & 6.06 & 5.23 \\
\hline 3 & 9.495058 & 7.990805 & 9.49 & 7.99 \\
\hline 4 & 12.300544 & 10.277019 & 12.29 & 10.27 \\
\hline 5 & 14.375397 & 12.113396 & 14.28 & 12.11 \\
\hline 6 & 16.168130 & 13.535662 & & 13.51 \\
\hline 7 & 18.343813 & 14.647713 & & 14.48 \\
\hline 8 & 20.916405 & 15.694926 & & 15.00 \\
\hline 9 & 23.816396 & 16.893204 & & \\
\hline 10 & 27.002109 & 18.303307 & & \\
\hline
\end{tabular}

tool for future studies. Furthermore, we contend that the offered nonlinear model existence of metastable kinkantikink bound states for the function of spin density on a two-dimensional graphene surface is possible. Finally, the numerical calculations show that the interval change between the next levels generally decreases with the energy growth, and since some value of energy, the spectrum becomes continuous.

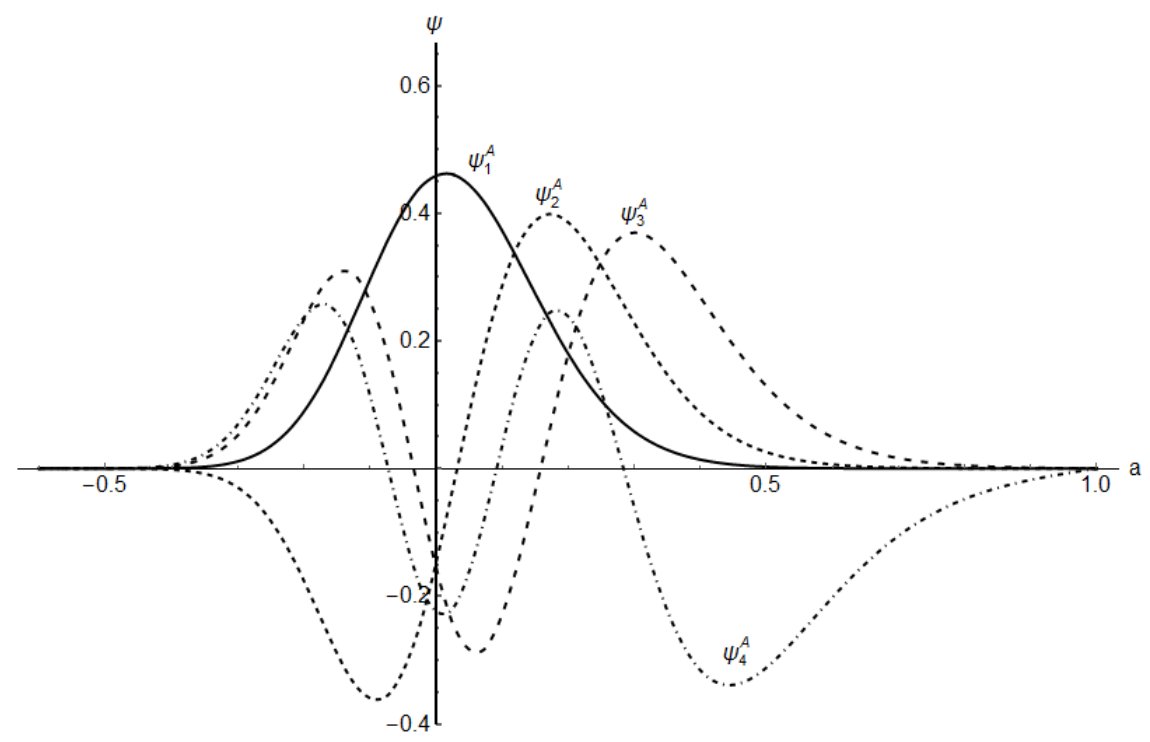

FIG. 2. First four eigenfunctions of model A with $s_{0}=2$ and $\lambda=1$

\section{Conclusion}

We proposed a reasonable numerical model which offered approximate solutions for the spin density's distribution of the stationary pseudo-spin waves on the surface of the graphene monoatomic film. We have obtained the discrete energy spectra and the corresponding eigenfunctions of nonlinear spin waves in the graphene structure. 


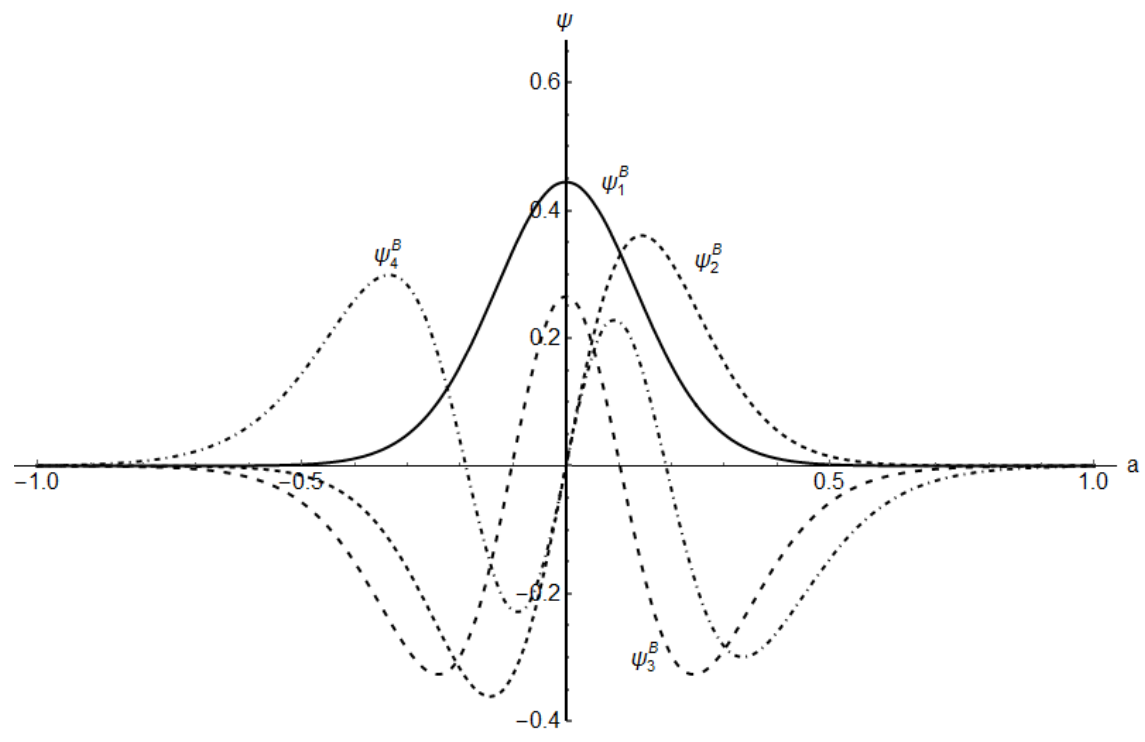

FIG. 3. First four eigenfunctions of model B with $s_{0}=2$ and $\lambda=1$

More complete numerical results may be obtained by the Chebyshev pseudospectral method. These results may be applied to create the nonlinear spin waves in the graphene structures.

\section{Acknowledgements}

The author is greatly indebted to Prof. D. S. Kulaybov and to K. P. Lovetskiy for suggesting the problem and for many stimulating conversations.

The publication was prepared with the support of the RUDN University Program 5-100.

\section{References}

[1] Grachev D.D., Sevastianov L.A., Lovetskiy K.P. Nonlinear spin waves in graphene structures. SPIN, 2014, 4(3), P. 1-13.

[2] Grachev D.D., Sevastianov L.A. Method of spin wave formation. Patent of Russia No. 2477907, 2010,7 pp.

[3] Grachev D.D., Sevastianov L.A. Method for generating spin waves. Patent of United States No. US8,779,765 B2, 2014,6 pp.

[4] Grachev D.D., Rybakov Y.P., Sevastianov L.A., Sheka E.F. Ferromagnetism in graphen and fulleren nanostructures. Theory, modelling, experiment. RUDN J. of $M I P h, 2010,1$, P. 20-27.

[5] Grachev D.D., Sevastianov L.A. Quantum field approach to the ferromagnetic properties of the graphene films. Nanostuctures. Math. phys. Model., 2011, 4(1), P. 5-15.

[6] Arzumanyan G.M., Ayrjan E.A., Grachev D.D., Sevastianov L.A. Quantum field model for graphene magnetism, modern trends in nanoscience. In book Balasoiu M., Arzumanyan G. M. Modern trends in nanoscience, Editura Academiei Romane, 2013,236 pp.

[7] Grachev D.D., Sevastianov L.A., Lovetskiy K.P., Gusev A.A., Vinitsky S.I., Derbov V.L. Model for spin waves and lasing in monolayer graphene films. Proc. of the SPIE, 2015, 9448, ID. $94481 \mathrm{~W}, 14 \mathrm{pp}$.

[8] Kulyabov D.S., Lovetskiy K.P., Nhat L.A. Simple model of nonlinear spin waves in graphene structures. RUDN J. of $M I P h ., 2018,26(3)$, P. 244-251.

[9] Roldan R., Fuchs J.-N., Goerbig M.O. Spin-flip excitations, spin waves, and magneto-excitons in graphene Landau levels at integer filling factors. Phys. Rev. B, 2010, 82, ID 205418, 13 pp.

[10] Culchac F.J., Latge A., Costa A.T. Spin waves in graphene nanoribbon devices. Phys. Rev. B, 2012,86 ID: 115407,11 pp.

[11] Culchac F.J., Latge A., Costa A. T. Spin waves in zigzag graphene nanoribbons and the stability of edge ferromagnetism. New J. Phys., 2011, 13, ID: 033028, 5 pp.

[12] Matthew A., Carsten U. Collective charge and spin excitations in graphene with in-plane magnetic fields. In Abstracts of the Conference APS March Meeting, New Orleans - Louisiana, March 13, 2017, 64(4), Abstract ID: BAPS.2017.MAR.P42.9, P. 1024.

[13] Mason J.C., Handscomb D.C. Chebyshev polynomials. CRC Press LLC, 2003, 335 pp.

[14] Don W.S., Solomonoff A. Accuracy and speed in computing the Chebyshev collocation derivative. SIAM J. Sci. Comput., 1991, 16(6), P. $1253-1268$.

[15] Nhat L.A. Pseudospectral methods for nonlinear pendulum equations. Zh. Sib. Fed. Univ. Mat. Fiz., 2019, 12(1), P. 79-84.

[16] Martha L.A., James P.B. Differential equations with mathematica, ed. 3rd. Elsevier Inc, 2004,893 pp.

[17] Weideman J.A.C., Trefethen L.N. The eigenvalues of second-order spectral differentiation matrices. SIAM J. Numer. Analys., 1988, 25(6), P. $1279-1298$. 